\title{
Luis Felipe Martorell-Dávila 1909-2001 Biografía, Patronimios y Lista de Publicaciones
}

\author{
Silverio Medina-Gaud ${ }^{1}$ \\ J. Agric. Univ. P.R. 86(1-2):85-94 (2002)
}

Luis Felipe Martorell Dávila (Figura 1), el primer puertorriqueño en obtener un doctorado en entomología, murió el 18 de diciembre de 2001 en San Juan, Puerto Rico. Le sobreviven su esposa, la Sra. Mercedes (Nina) Castro, su hija, Lilliana (Lilly) y su nieto, Germán.

Nació en Yabucoa en el año 1909. Cursó estudios de escuela superior en la ciudad de Humacao, graduándose en 1928. Obtuvo su grado de bachiller en Ciencias Agrícolas en el Recinto Universitario de Mayagüez (antes Colegio de Agricultura y Artes Mecánicas) en 1932. En septiembre de ese año aceptó un puesto como agrónomo en el Ministerio de Salubridad, Agricultura y Cría, en la ciudad de Caracas, Venezuela, donde dictó cursos en zoología y entomología. En ese país ocupó más tarde la posición de Subdirector de la Escuela de Expertos Agropecuarios, en la ciudad de Maracay. Allí realizó investigaciones en los insectos del cacao y caña de azúcar. Durante su estadía en dicho país aprovechó parte de su tiempo para recolectar una extensa variedad de insectos. Esta colección le sirvió de base para su tesis de grado de Maestro en Ciencias con especialización en Entomología, grado que obtuvo en el 1934 en la Universidad Estatal de Ohio, en Columbus, Ohio. Posteriormente publicó varios manuscritos sobre la tesis $(7,8)$.

En junio de 1934, tras regresar a Puerto Rico, fue nombrado Director de la Sección de Entomología de la "Puerto Rico Emergency Relief Administration" (PRERA) en San Juan, donde realizó investigaciones sobre los insectos de los vegetales (2). En septiembre del mismo año pasó a ocupar el puesto de técnico en el Servicio Forestal Federal y más tarde el de "Junior Forester" y Ayudante del Silvicultor hasta septiembre de 1936. Ese mismo mes pasó a ocupar el puesto de Entomólogo Ayudante en la Estación Experimental Agrícola (EEA). En el 1943 fue ascendido al puesto de Entomólogo Asociado, y en el 1950 al de Entomólogo. Fue Director del Departamento de Entomología desde 1953

\footnotetext{
${ }^{1}$ Entomólogo Ad-Honorem, Dept. Protección de Cultivos, Estación Experimental Agrícola, Apartado Postal 21360, San Juan, P.R. 00928.
} 


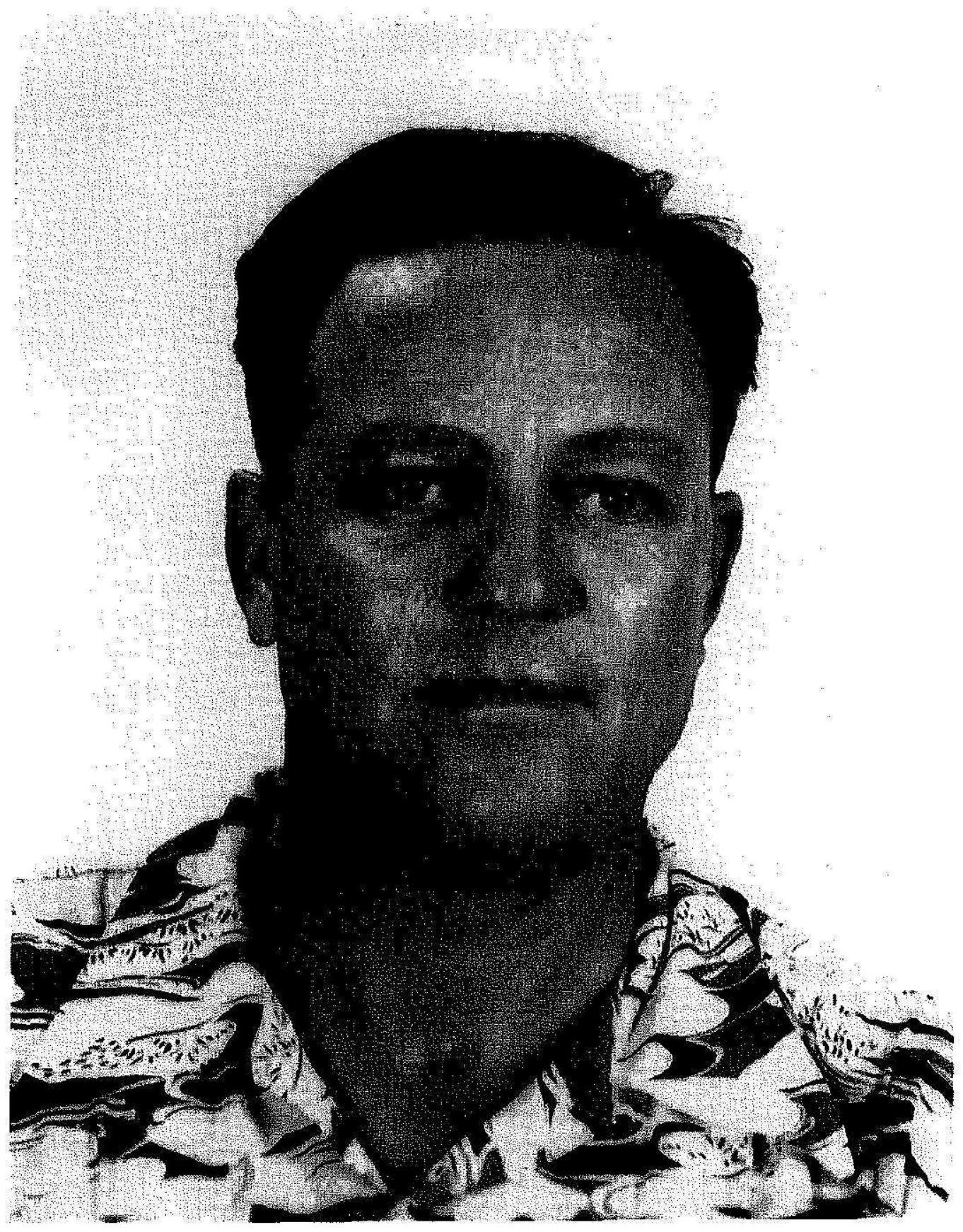

hasta 1969, cuando volvió a fungir como entomólogo hasta su jubilación en el 1972.

Entre los años 1934 y 1941, con la colaboración del Servicio Forestal, para transportación y alojamiento en sus campamentos forestales establecidos en la isla, hizo investigaciones sobre los insectos de los bosques, las cuales utilizó para su tesis doctoral. Para ese tiempo obtuvo 
un gran conocimiento sobre la flora y fauna de Puerto Rico $(9,10,11$, $12,13,15,16,17,20)$. Simultáneamente durante los años del $1940 \mathrm{al}$ 1942 se matriculó en trabajos de investigación fuera del recinto en la Universidad Estatal de Ohio. Retornó a Ohio en el verano del 1942, después de dos años de matrícula y presentación de informes trimestrales a su consejero en dicha universidad. En el verano del 1943, regresó a Puerto Rico con el grado de Doctor en Filosofía con especialización en Entomología, siendo el primer puertorriqueño en obtener ese grado en este campo de la ciencia.

De 1936 al 1941 llevó a cabo investigaciones intensivas en las plagas que atacan el cultivo de la caña de azúcar en Puerto Rico. En colaboración con el Dr. George N. Wolcott, Entomólogo del Departamento de Entomología de la EEA por más de 30 años, publicó una serie de artículos sobre este cultivo $(4,18,19,21,22)$. Siempre mantuvo gran interés en las investigaciones entomológicas relacionadas con la caña de azúcar, publicando posteriormente una serie de trabajos científicos, algunos en colaboración con sus compañeros de investigación (34, 35, $44,51,52,53,54,55,56,57)$.

Del 1952 al 1956 fue asesor técnico de la "Commonwealth Pest Control Corporation", firma dedicada al control de plagas caseras e industriales; con oficinas principales en Pittsburg, Pennsylvania, y con oficina en San Juan. En el 1961 fue también asesor técnico de la Agencia Internacional de Desarrollo (AID) del Departamento de Estado de los Estados Unidos de América, realizando investigaciones entomológicas de la caña de azúcar en la zona de Santa Cruz, Bolivia.

Realizó viajes oficiales relacionados con investigaciones entomológicas a las islas de San Tomás, Santa Cruz, Martinica, Guadalupe, Barbados y Trinidad. También visitó Guyana Inglesa, Brasil, Venezuela, Bolivia, República Dominicana, Haití, Jamaica, Cuba, México y a los estados de Florida, Carolina del Norte, Ohio, Louisiana y Texas de los Estados Unidos de América.

Durante los años del 1950 al 1953 y del 1961 al 1964, con la colaboración del Dr. Ishver Bangdiwala, Biómetra de la EEA, analizó los datos de campo obtenidos de los catastros de los daños producidos por la oruga barreno de la caña de azúcar, Diatraea saccharalis, y las ratas, Rattus spp., en Puerto Rico. Estas investigaciones fueron efectuadas a través de varios años con la colaboración de Silverio Medina Gaud, Rafael Bonilla y José C. García Tudurí. Los resultados finales fueron presentados en reuniones científicas internacionales llevadas a cabo en los Congresos de Tecnólogos Azucareros celebrados en 1955 y 1965, en Barbados y San Juan, respectivamente (34, 35, 36, 37, 52, 53, 54, 55, 56).

Siempre mantuvo un gran interés y entusiasmo en conocer más sobre la fauna entomológica de Puerto Rico. Bajo su liderazgo logró que reco- 
nocidos entomólogos de diferentes universidades de Estados Unidos de América, del Museo de Historia Nacional de Washington y el de Historia Natural de Nueva York, visitaran Puerto Rico para estudiar insectos y artrópodos de importancia económica, dando así a conocer nuestra fauna. Además de su interés en la taxonomía general de los insectos, participó en la introducción de parásitos para control biológico. Como puertorriqueño fue un pionero al realizar viajes de estudio a Brasil en búsqueda de parásitos para el combate efectivo de la changa en Puerto Rico. Realizó estudios con insecticidas para el control de las plagas de los cultivos de importancia económica en Puerto Rico: caña de azúcar, plátanos y guineos, batata, café, tabaco y algodón, y para el tratamiento y protección de las maderas en contra del comején y otras plagas.

En colaboración con el Dr. John S. Caldwell, Entomólogo norteamericano y compañero de estudios en la Universidad Estatal de Ohio, trabajó dos años (1947 y 1948) haciendo un catastro y estudiando los Homópteros de Puerto Rico e islas adyacentes. Este trabajo culminó con varias publicaciones taxonómicas y biológicas sobre los Homópteros Auchenorhincos de Puerto Rico $(26,27,29)$.

Durante los años del 1960 al 1970 colaboró en el estudio de los áfidos de Puerto Rico con el Dr. Clyde F. Smith, Afidiólogo y exdirector del Departamento de Entomología de la Universidad Estatal de Carolina del Norte, y con los doctores Mario E. Pérez y Silverio Medina Gaud. Como resultado se publicó una monografía sobre los áfidos (Homoptera:Aphididae) de Puerto Rico $(50,64)$. Además, se publicaron otros artículos relacionados (42).

Su interés más allá de la entomología lo llevó a tomar talleres sobre nematología agrícola en los Estados Unidos de América, logrando así establecer exitosamente la Sección de Nematología dentro del Departamento de Entomología en la EEA de Río Piedras (quizás la primera en todo el Caribe, Centro y Sur América).

En colaboración con los científicos Roy Woodbury, Silverio Medina Gaud y José García Tudurí (Fitotaxónomo, Entomólogo y Auxiliar de Entomología, respectivamente) efectuó catastros periódicos en casi todo Puerto Rico, coleccionando plantas e insectos. Junto a García Tudurí coleccionó comejenes (Isoptera) y plantas en las islas y cayos adyacentes a Puerto Rico $(60,61,72,73,78,86)$. Todos los especímenes recogidos pasaron a ser parte del herbario y museo. Por su interés en la taxonomía botánica y entomológica logró mantener, aumentar y preservar las colecciones botánicas (Herbario) y la de insectos (Museo) en las que él ya colaboraba con el Dr. Wolcott. Antes de jubilarse publicó el Catálogo de las plantas hospederas de los insectos de Puerto Rico (84). Para fines de la década del 1980, el herbario, que él cuidó y aumentó con tanto esmero con la colaboración de Woodbury, Medina Gaud y García 
Tudurí, pasó mediante un acuerdo administrativo al Jardín Botánico de la Universidad de Puerto Rico.

En el 1971 publicó el primer estudio sobre la flora de la isla de Desecheo (65) y más tarde (1977) publicó el de la flora de las islas de Mona y Monito (85). También en el 1981 revisó y publicó con la colaboración de R. Woodbury y Allan Liogier el libro sobre los nombres vulgares y científicos de las plantas de Puerto Rico (86).

Durante su vida profesional realizó en la República Dominicana trabajos consistentes en catastros y asesoramiento técnico sobre los insectos del cacao, arroz, caña de azúcar y salud pública. En el 1972, después de retirado de la EEA, regresó a la República Dominicana donde trabajó en el Consejo Estatal de Azúcar realizando catastros y dando asesoramiento sobre los insectos de la caña de azúcar $(74,75$, 76). En los otoños del 1978 y 1979 visitó la Provincia de Jujuy, al norte de Argentina, donde hizo investigaciones sobre el control de los gusanos del suelo en caña de azúcar obteniendo exitoso control de estos insectos. En el 1978 al regresar a Puerto Rico, comenzó a trabajar en el Jardín Botánico de UPR, donde continuó, junto al Dr. Liogier, su trabajo taxonómico de la flora de Puerto Rico. En el 1982, en colaboración con el Dr. Liogier, publicó la primera lista taxonómica de la flora de Puerto Rico (87) la cual fue revisada en el 2000 (92).

El Dr. Martorell Dávila fue miembro de varias sociedades científicas y profesionales: "American Association for the Advancement of Science", Sociedad de Tecnólogos Azucareros, "Entomological Society of America", Sociedad Honorífica de Agrónomos Gamma Sigma Delta" y Asociación Americana de Fitotécnia de Puerto Rico. Fue socio fundador de la Sociedad Entomológica de Puerto Rico y miembro de la Sociedad Puertorriqueña de Ciencias Agrícolas.

Por su continuo interés en la entomología y su colaboración con otros entomólogos fue honrado con las siguientes designaciones de su nombre a un género, 11 especies de insectos y una especie de ácaro, nuevos para la ciencia. Estos son: Martorella puertoricensis Caldwell (Homoptera: Achilidae); Ceropsylla martorelli Caldwell (Homoptera: Psylliidae); Euceropsylla martorelli (Caldwell) (Homoptera: Psylliidae); Macrosiphum (Sitobion) martorelli C. F. Smith (Homoptera: Aphididae); Pintalia martorelli Caldwell (Homoptera: Cixiidae); Clastoptera martorelli Ramos (Homoptera: Cercopidae); Empoasca martorelli Metcalf (Homoptera: Cicadellidae); Amblycerus martorelli Bridwell (Coleoptera: Bruchidae); Phloeonemus martorelli Fisher (Coleoptera Colydiidae); Hydroptila martorelli Flint (Trichoptera: Hydroptilidae); Ocyptamus martorelli Telford (Diptera: Syrphidae); Borinquenula martorelli Walker \& Gurney (Orthoptera: Tettigoniidae) y Steneotarsonemus martorelli Cromroy (Acarina: Tarsonemidae). 
Durante su productiva vida publicó unas 93 publicaciones, algunas con sus colegas. Desde el 1961 hasta el 1972 fue editor de la página de jardinería en el periódico El Mundo en San Juan, Puerto Rico, donde publicó cerca de 450 artículos sobre horticultura, entomología, botánica, zoología y jardinería en general. Por esta gran labor educativa recibió en el 1968 el Premio de Periodismo del Instituto de Literatura Puertorriqueña.

Como reconocimiento a la excelente labor realizada por este distinguido científico, la Universidad de Puerto Rico le otorgó en el 1972 el título de Profesor Emérito.

\section{PUBLICACIONES}

1. Martorell, L. F., 1933. Informe de un viaje relacionado con investigaciones agrícolas en el Estado de Miranda, Venezuela, Memorias del Ministerio de Salubridad, Agricultura y Cría pp. 80-96.

2. Martorell, L. F., 1934. El Control de los Insectos en Vegetales y Cultivos Menores. Boletín No. 1, Sección de Entomología, PRERA, San Juan, PR.

3. Wolcott, G. N. y L. F. Martorell, 1937. Two insects new to Puerto Rico; the lycid beetle, Thonalmus chevrolati Bourgeois and the ephydrid fly Ephydra gracilis Packard. J. Agric. Univ. P.R. 21(4):535-538.

4. Wolcott, G. N. y L. F. Martorell, 1937. The ant Monomorium carbonarium Forel in a new role; as predator on the egg-clusters of Diatraea saccharalis Fabricius in Puerto Rican cane fields. J. Agric. Univ. P.R. 21(4):577-579.

5. Martorell, L. F., 1939. Methods of collecting and shipping Larra americana Saussure; a parasite of the Puerto Rican mole-cricket. Ann. Entomol. Soc. Amer., 32(4):703-712.

6. Martorell, L. F. y G. N. Wolcott, 1939, Forest tree insects. Ann. Rept., Agric. Exp. Sta., Río Piedras, PR. 1937-38, p. 44.

7. Martorell, L. F., 1939. Insects of the Aragua Valley, Venezuela, S.A. J. Agric. Univ. P.R. 23(4):177-232.

8. Martorell, L. F. y A. Escalona Sales 1939. Additional insect records from Venezuela. J. Agric. Univ. P.R. 23(4):233-264.

9. Martorell, L. F., 1939. Some notes on forest entomology. Carib. For: 1(1):25-26.

10. Martorell, L. F., 1940. Notes on the biology of Mesocondyla concordalis Hübner and its parasites. Carib. For. 1(1):18-19.

11. Martorell, L. F., 1940. Some notes on forest entomology. Carib. For: 1(2):31-32.

12. Martorell, L. F., 1940. Some notes on forest entomology. Carib. For 1(3):23-24.

13. Wolcott, G. N. y L. F. Martorell, 1940. Epidemics of fungus disease control insect pests in Puerto Rico. J. Econ. Entomol. 23(1):201-202.

14. Wolcott, G. N. y L. F. Martorell, 1940. Leafhopper reaction to lawn sprinkling. J. Econ. Entomol. 23:584.

15. Martorell, L. F., 1941. Some notes on forest entomology IV. Carib. For: 2(2):80-83.

16. Martorell, L. F., 1941. Biological notes on the sea-grape sawfly, Schizocera krugii Cresson in Puerto Rico. Carib. For. 2(3):141-144.

17. Martorell, L. F., 1941. Caculos y vaquitas en plantas ornamentales (May-beetles and curculios on ornamental plants). Boletín Trimestral, Est. Exp. Agr., UPR. Agricultura Experimental, Vol. 1(4):10.

18. Wolcott, G. N. y L. F. Martorell, 1942. The accidental introduction of a beneficial insect into Puerto Rico. Carib. For. 3(2):58-62 
19. Wolcott, G. N. y L. F. Martorell, 1943. Control of the sugarcane borer in Puerto Rico by laboratory reared parasites. J. Econ. Entomol. 36(3):486.

20. Martorell, L. F, 1943. Forests and forest entomology. Carib. For, 4(3):132-134.0

21. Wolcott, G. N. y L. F. Martorell, 1944. Natural parasitism by Trichogramma minutum of the eggs of the sugarcane moth borer, Diatraea saccharalis, in the cane fields of Puerto Rico. J. Agric. Univ. P.R. 27(2):39-83, fig. 12, ref.

22. Wolcott, G. N. y L. F. Martorell, 1944. The seasonal cycle of insect abundance in Puerto Rican cane fields. J. Agric. Univ. P.R. 27(2):85-114.

23. Wolcott, G. N. y L. F. Martorell, 1944. Las posibilidades de combatir el barreno de la caña de azúcar, Diatraea saccharalis Fabricius, en Puerto Rico llevando a los cañaverales los parásitos Trichogramma minutum Riley criados en laboratorios. Est. Exp. Agr., Río Piedras, Bol. 64: 1-16, pl. 1, tab. 2.

24. Wolcott, G. N. y L. F. Martorell, 1944. Introduced lady beetles on Mona Island. J. Econ. Entomol., 37(3):451-452.

25. Martorell, L. F., 1945. A survey of the forest insects of Puerto Rico. Part I \& II. J. Agric. Univ. P.R. 29 (3-4):69-608.

26. Caldwell, J. S. y L. F. Martorell, 1950. Review of the Auchenorynchous Homoptera of Puerto Rico, Part I, Cicadellidae. J. Agric. Univ. P.R. 34(1):1-132.

27. Caldwell, J. S. y L. F. Martorell, 1950. Review of the Auchenorhynchous Homoptera of Puerto Rico, Part II, The Fulgoroidea, except Kinnaridae. J. Agric. Univ. P.R. 34(2):133-269.

28. Martorell, L. F., 1951. Cotton pink bollworm in Puerto Rico. Agric. Exp. Sta., Univ. P.R. Bull. 93:1-40.

29. Caldwell, J. S. y L. F. Martorell, 1951. A brief review of the Psyllidae of Puerto Rico (Homoptera). Ann. Entomol. Soc. Amer. 44(4):603-613.

30. Caldwell, J. S. y L. T. Martorell, 1952. New leafhoppers from Puerto Rico. J. Agric. Univ. P.R. 35(2): 88-89.

31. Martorell, L. F. y J. Adsuar, 1952. Insects associated with papaya virus diseases in the Antilles and Florida. J. Agric. Univ. P.R. 36(4):319-329. (Also in J. Econ. Entomol. 45(6):861-869).

32. Hernández Medina, E., L. F. Martorell y G. N. Wolcott, 1953. The effects of winddrift of weed-killer on some Puerto Rican trees. Science 118(3055):74-75.

33. Martorell, L. F., 1953. Qué árbol sembraré. Carib. For. 14(3-4):152-160.

34. Martorell, L. F. y C. H. Burleigh, 1954. Ineffectiveness of the overhead irrigation method for application of insecticides to control the sugar-cane moth-borer Diatraea saccharalis (Fabricius). J. Agric. Univ. P.R. 38(1):38-60.

35. Martorell, L. F. y J. S. Bangdiwala, 1954. Sucrose content of sugar cane as affected by moth-borer, Diatraea saccharalis (Fabricius), infestation. J. Agric. Univ. P.R. $38(1): 22-37$.

36. Bangdiwala, I. S. y L. F. Martorell, 1954. Correlation between stalk and joint infestation by sugar-cane moth-borer, Diatraea saccharalis (Fabricius). Proc. 8th Congress Int. Soc. Sugar-cane Technol., pp. 593-601.

37. Martorell, L. F. y I. S. Bangdiwala, 1954. Sucrose content of sugarcane as affected by moth-borer (Diatraea saccharalis) infestation. Proc. 8th Congress Int. Soc. Sugar Cane Technol., pp. 602-614.

38. Martorell, L. F, 1955. Acanthochila spinacosta Van Duzee, a new tingitid for Puerto Rico, J. Agric. Univ. P.R. 39(1):47-48.

39. Pastor Rodríguez, J. y L. F. Martorell, 1956. El cultivo del algodón en Puerto Rico. Est. Exp. Agric., Bol. 131:1-102.

40. Samuels, G., H. Cibes Viade y L. F. Martorell, 1956. Increased use of agricultural chemicals in Puerto Rico. Sugar 51(10):38-41. 
41. Rodríguez, J. P. y L. F. Martorell, 1957. El cultivo del algodón. La Hacienda 54(4):26-31, 36.

42. Smith, C. F., L. F. Martorell y M. E. Pérez Escolar, 1958. Myzus persicae (Sulzer) in Puerto Rico. J. Agric, Univ. P.R., 42(4):263-266.

43. Martorell, L. F., 1959. Preliminary report on orchid pests in Puerto Rico. The Orchid Soc. P.R., Ann. Prog., pp. 19-27.

44. Martorell, L. F., 1959. Control of the important sugarcane insect pests in Puerto Rico. Sugar J. 22(1):55-63.

45. Martorell, L. F., 1959. Preliminary report on orchid pests in Puerto Rico. Agric. Exp. Sta., Univ. P.R., Misc. Publ. 25.

46. Kramer, J. P. y L. F. Martorell, 1960. First records for the rice plant hopper, Sogata orizicola Muir, in Puerto Rico (Homoptera: Fulgoridae: Delphacidae) J. Agric. Univ. P.R. 44(4):163-165.

47. Martorell, L. F., 1961. Plagas comunes de los jardines y su control. Est. Exp. Agric., U.P.R. Publ. Misc, 36.

48. Martorell, L. F., 1961. Plagas comunes en los jardines y su control. Rev. Agric. P.R. 48(1):214-220.

49. Martorell, L. F., 1962. Árboles ornamentales. Rev. Agric. P.R., 49(1):

50. Smith, C. F., L. F. Martorell y M. E. Pérez Escolar, 1963. The Aphididae of Puerto Rico. Agric. Exp. Sta., Univ. P.R., Tech. Paper 37:1-121.

51. Martorell, L. F., 1966. Field practices used to control sugar cane pests in Puerto Rico. (In English and Spanish Sugar y Azúcar. 61(4): 28-31 and 53-57.

52. Martorell, L. F. y S. Medina Gaud, 1967. Status of important insect pests of sugarcane in Puerto Rico and their control. 12th Congress Int. Soc. Sugarcane Tech., San Juan, P.R., April 1965, Elsevier Publ. Co., pp. 1278-1286.

53. Martorell, L. F. y S. Medina Gaud, 1967. Notes on parasitism of the sugarcane moth borer, Diatraea saccharalis (Fabricius) in Puerto Rican cane fields. 12th. Congress Int. Soc. Sugarcane Tech., San Juan, P.R., April 1965, Elsevier Publ. Co., pp. 1295-1303.

54. Medina Gaud, S., L. F. Martorell y R. Bonilla, 1967. Notes on the biology and control of the yellow aphid of sugarcane, Sipha flava (Forbes) in Puerto Rico. 12th Congress Int. Soc. Sugarcane Tech., San Juan, P.R., April 1965, Elsevier Publ. Co., pp. 1307-1320.

55. Bangdiwala, I. S., L. F. Martorell, S. Medina Gaud y A. Cruz Miret, 1967. Further studies in correlation between stalk and joint infestation by sugarcane moth-borer, Diatraea saccharalis (Fabricius) in Puerto Rico. 12th Congress Int. Soc. Sugarcane Tech., San Juan, P.R., April 1965, Elsevier Publ. Co., pp. 1373-1382.

56. Martorell, L. F., S. Medina Gaud y A. Cruz Miret, 1967. A preliminary report on rat damage in Puerto Rican fields. 12th Congress Int. Soc. Sugarcane Tech., San Juan, P.R., April 1965, Elsevier Publ. Co., pp. 1435-1443.

57. Martorell, L. F., 1968. Combate de plagas de la caña en Puerto Rico. Publ. FA-65. Serv. Ext. Agric., Univ. P.R.

58. Muriente, J. E., L. F. Martorell y M. E. Pérez Escolar, 1968. La chinche de los pastos. Pub. Misc. Serv. Ext. Agric. 5M FA 63:1-5.

59. Hensley, S. D. y L. F. Martorell, 1969. Comparison of growth and development of sugarcane borer larvae from Puerto Rico and Louisiana. J. Agric. Univ. P.R. $53(2): 147-148$.

60. García Tudurí, J, C., L. F. Martorell y S. Medina Gaud, 1971. Geographical distribution and host plants of the cactus moth, Cactoblastis cactorum (Berg) in Puerto Rico and the United States Virgin Islands. J. Agric. Univ. P.R. 55(1):130-134.

61. Martorell, L. F. y J. C. García Tudurí, 1971. New locality and host plant records for two species of Nasutitermes (Isoptera-Termitidae). J. Agric. Univ. P.R. 55(1):135137. 
62. Martorell, L. F., S. Medina Gaud y E. Jordán Musa, 1971. Indexes to the Journal of Agriculture of the University of Puerto Rico, Volumes 1-47 (1917-1963), J. Agric. Univ. P.R. 55(3):275-357.

63. Martorell, L. F., 1971. Indexes to the Journal of Agriculture of the University of Puerto Rico. Vol. 48-52 (1964-1968)-Supplement No. 1. J. Agric. Univ. P.R. 55(3):358-385.

64. Smith, C. F., S. Medina Gaud, L. F. Martorell y M. E. Pérez Escolar, 1971. Additions and corrections to the Aphididae of Puerto Rico. J. Agric. Univ. P.R. 55(2):192-258.

65. Woodbury, R. C., L. F. Martorell y J. C. García Tudurí, 1971. The flora of Desecheo Island, Puerto Rico. J. Agric. Univ. P.R. 55(4):478-505.

66. Medina Gaud, S. y L. F. Martorell, 1971. Indexes to the Journal of the University of Puerto Rico, volumes 53-55 (1969-1971), Supplement No. 2. J. Agric. Univ. P.R. 55(4): 520-544.

67. Martorell, L. F., 1961-1972. Más de 450 artículos sobre Horticultura, Entomología, Botánica, Zoología y Jardinería en General, publicados en la Página de Jardinería en el periódico El Mundo, San Juan, PR. en el período comprendido entre febrero 1961 y febrero 1972.

68. Maramorosch, K., L. F. Martorell, J. Bird y P. L. Meléndez, 1972. Platypus rugulosus (Platypodidae) and Xyleborus ferrugineus (Scolytidae) and certain diseases of coconut palms in Puerto Rico. J. New York Entomol. Soc. 80(4):238-240.

69. Medina Gaud, S. y L. F. Martorell, 1973. New insect records for Puerto Rico. J. Agric. Univ. P.R. 57(3):247-254.

70. Martorell, L. F. y J. C. García Tudurí, 1973. Notes on the accidental introduction of Umbonia crassicornis (Amyot \& Serville) (Homoptera: Membracidae) into Puerto Rico. J. Agric. Univ. P.R. 57(4):307-313.

71. Liu, L. J. y L. F. Martorell, 1973. Diplodia stem canker and die-back of Casuarina equisetifolia in Puerto Rico. J. Agric. Univ. P.R. 57(3):255-35.

72. MartorelI, L. F., 1973. Glyptotermes liberatus (Snyder) (Isoptera-Kalotermitidae) a new termite record for Puerto Rico. J. Agric. Univ. P.R. 57(4):355-356.

73. Martorell, L. F, 1973. The insects of Mona Island, Puerto Rico. In: Mona and Monito Islands: an assessment of their natural and historical resources. Vol. 2. App J. Pp. J.1-J.-7. Junta de Calidad Ambiental (Env. Qual. Bd.). San Juan, PR.

74. Martorell, L. F., J. A. Burgos y F. Mejía Biaggi, 1973. Preliminary investigations on the sugarcane insects of the Dominican Republic, Proc. of the 1973 Meeting of the W.I. Sugarcane Technol., pp. 247-277, Barbados.

75. Burgos, J. A. y L. F. Martorell, 1973. The Calisto (Lepidoptera-Satyridae) problem in sugarcane in the Dominican Republic, Proc. of the 1973 Meeting of W.I. Sugarcane Technol. pp. 272-277, Barbados.

76. Mejía Biaggi, F. y L. F. Martorell, 1973. Notes one the sugarcane soil insect pests in the Dominican Republic. Proc. of the 1973 Meeting of the W.I. Sugarcane Technol., pp. 278-281, Barbados.

77. Martorell, L. F. y S. Medina Gaud, 1974. Orchid pests in Puerto Rico. J. Agric. Univ. P.R. 58(1):105-124.

78. García Tudurí, J., S. Medina Gaud y L. F. Martorell, 1974. Preliminary list of the insects of Desecheo Island, Puerto Rico. J. Agric. Univ. P.R. 58(1):125-133.

79. Medina Gaud, S. y L. F. Martorell, 1974. The insects of Caja de Muertos Island, Puerto Rico. J. Agric. Univ. P.R. 58(2):244-272.

80. Martorell, L. F., 1974. La viuda negra. Est. Exp. Agric., Univ. P.R., Nota Informativa Núm. 155:1-2.

81. Martorell, L. F. y J. C. García Tudurí, 1974. La chinche espinosa y su control en Puerto Rico. Est. Exp. Agric. Univ. P.R. Adelantos Científicos 6:1-2. 
82. Medina Gaud, S., J. C. García Tudurí, L. F. Martorell y J. Rodríguez, 1974. Posibles sustitutos del Aldrín y el Dieldrin para el control del gorgojo del guineo y el plátano. Adelantos Científicos. Est. Exp. Agric. Univ. P.R., Núm. 11:1-2.

83. Medina Gaud, S., J. C. García Tudurí, L. F. Martorell y J. Rodríguez, 1974. Preliminary screening of pesticides for the control of the banana root borer. J. Agric. Univ. P.R. $59(1): 79-81$.

84. Martorell, L. F., 1976. Annotated food plant catalog of the insects of Puerto Rico. Agric. Exp. Sta. Univ. P.R., Dept. Entomol. pp. 1-303.

85. Woodbury, R. O., L. F. Martorell y J. C. García Tudurí, 1977. The flora of Mona and Monito Islands, Puerto Rico (West Indies). Agric. Exp. Sta., Univ. P.R. Bol. 252:1-60.

86. Martorell, L. F., A. H. Liogier y R. O. Woodbury, 1981. Catálogo de los nombres vulgares y científicos de las plantas de Puerto Rico. Est. Exp. Agric., Univ. P.R., Bol. 263:1-231.

87. Liogier, H. A. y L. F. Martorell, 1982. Flora of Puerto Rico and adjacent islands: a systematic synopsis. 1st. Edition. Editorial Univ. P.R.; pp. 1-342.

88. Medina Gaud, S., L. F. Martorell y H. O'Farrill, 1983. Los roedores y su control en Puerto Rico. Univ. P.R., Serv. Ext. Agric., Río Piedras, Pub. 21:1-25.

89. Medina Gaud, S., H. O'Farrill y L. F. Martorell, 1987. Los roedores domésticos y su control en Puerto Rico. Univ. P.R., Serv. Ext. Agric., Mayagüez, PR., julio 1987, pp. 1-20.

90. Medina Gaud, S., L. F. Martorell y N. Díaz Acín, 1987. Los comejenes de importancia económica en Puerto Rico. Univ. P.R., Est. Exp. Agric., Río Piedras, Bol. 280:1-28.

91. Martorell, L. F., H. O'Farrill Nieves y S. Medina Gaud, 1991. Control de plagas en bosques. (Revisado). Univ. P.R., Mayagüez, Pub. Serv. Ext. Agric., pp. 1-27.

92. Liogier, H. A. y L. F. Martorell, 2000. Flora of Puerto Rico and adjacent islands: a systematic synopsis. 2nd edition, Editorial Univ. P.R.; pp. 1-382.

93. Medina Gaud, S., L. F. Martorell y J. Maldonado Capriles, 2002. Catálogo de los nombres comunes de los insectos y acarinos de importancia económica de Puerto Rico (En Imprenta).

Preparado por: Silverio Medina Gaud, Ph.D.

Enero 2002 